\title{
Videoların Derin Öğrenme ile Sınıflandırılarak Filtrelenmesi
}

\author{
Murat Kazanç ${ }^{1 *}$, Tolga Ensari², Mustafa Dağtekin ${ }^{3}$ \\ 1 İstanbul Üniversitesi - Cerrahpaşa, Mühendislik Fakültesi, Bilgisayar Mühendisliği, İstanbul Bölümü, Türkiye (ORCID: 0000-0002-8405-0181) \\ ${ }^{2}$ Arkansas Tech University, College of Engineering \& Applied Science, Dept of Computer and Information Science, Arkansas, USA (ORCID: 0000-0003-0896-3058) \\ ${ }^{3}$ İstanbul Üniversitesi - Cerrahpaşa, Mühendislik Fakültesi, Bilgisayar Mühendisliği Bölümü, İstanbul, Türkiye (ORCID :0000-0002-0797-9392)
}

(International Congress on Human-Computer Interaction, Optimization and Robotic Applications (HORA) 2021 - 11-13 June 2021)

(DOI: $10.31590 /$ ejosat.952481)

ATIF/REFERENCE: Kazanç, M., Ensari, T., \& Dağtekin, M. (2020). Videoların Derin Öğrenme ile Sınıflandırılarak Filtrelenmesi. Avrupa Bilim ve Teknoloji Dergisi, (26), 338-342.

\section{$\ddot{O} \mathbf{z}$}

Bu çalışmada derin öğrenme metodu olan evrişimli sinir ağları (Convolutional Neural Networks - CNN) ve transfer öğrenme metodu kullanılarak tütün mamulleri, alkollü içecek ve silah gibi istenmeyen nesnelerin tespitini ve sınıflamasını yapan bir model geliştirilmiştir. Bu model Tensorflow JS'e dönüştürülerek, internet tarayıcısı için bir eklenti olarak geliştirilmiştir. Bu eklenti ile izlenen videolardan anlık görüntüler alınarak eğitilen model üzerinde sınıflandırmalar yapılmıştır. Videolar üzerinde yapılan sınıflamalarda gerekli görülen sonuçlar, Google tarafindan sağlanan bir bulut hizmeti olan Firebase'in RealTimeDatabase' e kaydedilmiş̧ir. Kaydedilen veri tabanı kullanılarak daha önceden kötü içerik tespiti yapılmış videoların engellenmesi sağlanmıştır. Tarayıcıdan yapılan tespitlerin son 25 tanesi bilgilendirme amaçlı kullanıcı tarafından görüntülenebilmektedir. Bu çalışmada, izlenen videolardan anlık görüntüler alınarak model ile sınıflama yapılmıştır. Gerekli hallerde videonun bilgisi veri tabanına eklenebilmektedir ve veri tabanına kayıtlı videoların görüntülenmesi filtre edilebilmektedir. Geliştirilen sistem hem fiziksel cihazlar hem de emülatör aracıllğıyla test edilmiştir. CNN ile geliştirilen derin öğrenme modelinin ağ yapısı oluşturulmasında iki yol benimsenmiştir. Birincisinde, tüm ağ modeli tarafımızca oluşturduğumuz modeldir. Bu modelde, parametre sayıs1 7.752 .707 adettir ve $\% 86,75$ eğitim ve $\% 88,02$ test doğruluğu elde edilmiştir. İkinci olarak, transfer öğrenme metodu kullanılarak, başarısı literatürde kanıtlanmış modellerden olan MobileNetV2 tercih edilmiştir. Çıkış katmanları düzenlenmiş bu modelde eğitilebilir 593.155 adet ve toplamda 2.852 .675 adet parametre ile $\% 65,34$ eğitim ve $\% 50,35$ test doğruluğu elde edilmiş̧ir. Yapılan çalışma sonucunda, video içeriklerini filtrelemek için CNN modelinin daha verimli olacağ 1 bulgusuna ulaşılmıştır.

Anahtar Kelimeler: Evrişimli sinir ağları (CNN), derin öğrenme, video filtreleme, transfer öğrenme.

\section{Filtering Videos by Classification with Deep Learning}

\begin{abstract}
In this study, a model that detects and classifies unwanted objects such as tobacco products, alcoholic beverages and weapons was developed using Convolutional Neural Networks $(\mathrm{CNN})$, which is a deep learning methods and transfer learning. This model was converted to Tensorflow JS and developed as an add-on for an Internet Browser. With this add-on, snapshots were taken from the watched videos and classifications were made using the trained model. The results that are deemed necessary in the classification of the videos were recorded to Firebase's RealTimeDatabase, a cloud service provided by Google. By using the recorded database, videos that had previously been detected with harmful content were blocked. The last 25 of the definitions made from the browser can be viewed by the user for informational purposes. In this study, snapshots from watched videos were taken and classified with the model. If necessary, information about the video can be added to the database, and videos saved in the database can be filtered. The developed system has been tested through both physical devices and emulator. Two ways have been adopted in creating the network structure of the model developed with CNN. First, the entire network model is the one that is created by us. In this model, the number of parameters was $7,752,707$ and the accuracy of $84.84 \%$ training and $79.77 \%$ testing was achieved. Second, MobileNetV2, which is one of the models whose success has been proven in the literature, was preferred using the transfer learning method. With 593,155 trainable parameters and 2,852,675 parameters in total, $65.34 \%$ training and $50.35 \%$ test accuracy was achieved in this model with output layers arranged. As a result of the study, it was found that the CNN model would be more efficient in filtering video content.
\end{abstract}

Keywords: Convolutional neural networks (CNN), deep learning, video filtering, transfer learning.

\footnotetext{
* Sorumlu Yazar: İstanbul Üniversitesi - Cerrahpaşa, Mühendislik Fakültesi, Bilgisayar Mühendisliği Bölümü, İstanbul, Türkiye, ORCID: 00000002-8405-0181, murat.kazanc@,ogr.iuc.edu.tr
} 


\section{Giriş}

Derin öğrenme ham veriden kademeli olarak daha yüksek seviyeli özellikler çıkarmak için çoklu katmanlar kullanan bir makine öğrenmesi alt sınıfı olarak tanımlanmaktadır. Örneğin, görüntü sınıflandırmada alt katmanlar kenarları tanımlarken yüksek katmanlar rakam, harfler veya yüzler gibi kavramları tanımlayabilir. Bu çalışmada, derin öğrenme kullanılarak video sınıflandırma modeli geliştirilmiştir. Sınıflandırma için veri kümesindeki belirgin özelliklerinin ortaya çıkarılması gerekir. Bunun için evrişimli sinir ağları (convolutional neural networks) geliştirilmiştir. Böylece, özellik çıkarımı yapılabilmektedir. Örnek olarak eğriler, kenarlar gibi özelliklerin belirginleşmesi bu ağlar ile sağlanabilir (LeCun, Bengio, \& Hinton, 2015).

Tarayıcı eklentileri görüntülenen internet sitesinin içerisine, normalde sitenin içeriğinde olmayan yeni özellikler eklemek için kullanılabileceği gibi tarayıcıda bulunmayan özellikler eklemek için de kullanılmaktadır. Örneğin, tarayıcılar için web sitelerindeki reklamları engelleyen eklentiler yoğun bir şekilde kullanılmaktadır. Bu çalışmada, normalde görüntülenen sitenin içeriğinde olmayan javascript kodları eklenerek işlemler gerçekleştirilmiştir. Tarayıcı eklentisi, html sayfa içerisindeki video etiketini bularak oynatılan video içerisindeki görüntülerde sinıfların bulunmasidır. Yapılan tespitlerle bir bulut veri tabanında depolanarak bir katalog oluşturulmuştur. Ayrıca, HEVC (Yüksek verimli video kodlamasi-High Effiency Video Coding) kodlamasında video sıkıştırma işleminde evrişimli sinir ağlarının kullanılabileceği gösterilmiştir (Jia, ve diğerleri, 2019). Derin öğrenme ve Markov karar süreçleri kullanılarak IP protokolü üzerinden yayın akışı gerçekleştiren beş yayıncının (YouTube, YouTube TV, Netflix, Amazon Prime ve HBO) veri paketlerinin makine öğrenmesi kullanılarak sınıflandırılması da mümkündür. Benzer çalışmada, farklı hizmetlerden gelen benzer video akış trafiği verilerinin doğru bir şekilde sınıflandırılması sağlanmıştır (Shaout \& Crispi, 2020). Google Research tarafindan 8 milyon video kullanılarak 200 farklı etiket ile veri kümesi oluşturulmuştur. Bu veri kümesi araştırmacıların erişimine açıktır (Abu-El-Haija, ve diğerleri, 2016).

Videoları sınıflandırmak için meta verileri, arama motoru ve sosyal medya verileri değerlendirilmesi mümkündür. Videoyu sınıflandırırken kare analizi, ses ve metin verileri kullanmaktadır. Eğer video içerisinde alakasız, nefret, suça teşvik, yetişkin içerik varsa videonun yayınlanmasına izin verilmemektedir. Bunun için bağlam kullanılabilir (Hammam, 2019). Youtube benzeri ortamlarda görüntülenen videonun meta verilerine bakılması ve NLP metotları kullanılması makul görülmektedir. Video yüklendiği zaman otomatik olarak ön tanımlı kategorilerden birisine atanması ve ek açıklamalar oluşturulması önemli bir özellik olabilmektedir. Video sinıflanırken görsel, işitsel, açıklama, etiketler, başlık ve yorumlar dikkate alınmalıdır. $\mathrm{Bu}$ sayede, arama sonuçları iyileştirilmesi mümkün olacaktır (Chen, Chang, \& Yeh, 2017).

Derin öğrenme video sınıflaması için çözümler sunmaktadır. Son dönemde yapilan çalışmalarda, ikili ağ yapısı önerilmektedir. Birinci ağ yapısı, videonun tamamında hareketlerin algılanmasına odaklanırken, ikinci ağ ile videodan kare görüntüleri üzerinden nesnelerin tespit edilmesi sağlanmaktadır. Buradan hareketle, iki ağın sonuçlarından video içeriği anlaşılabilmektedir. Herhangi bir eylemin zaman ve mekânsal olarak anlaşılabilmesi için birkaç hareketin arka arkaya yapılması gerekmektedir. Bu sebeple, ek olarak hafiza özelliği olan model kullanılması performansı e-ISSN: 2148-2683 artıracaktır. Bununla ilgili bir diğer öneri ise denetimsiz öğrenme metotlarının video sınıflamada kullanılabileceği olmuştur. Veri kümesindeki videolar, denetimsiz öğrenme metotları ile ön gruplama yapıldıktan sonra asıl sisteme verilmesi ile detaylı sınıflandırma yapılabileceği ifade edilmektedir (Wu, Yao, Fu, \& Jiang, 2017). CNN kullanılarak videolar üzerinde sınıflandırma yapılması için model eğitimi uzun süre alabilmektedir (Karpathy, ve diğerleri, 2014). Video sinıflamada iyi bir performans elde etmek için büyük miktarda etiketli veri gerekmektedir. $\mathrm{Bu}$ bağımlılı̆̆ en aza indirmek için yarı denetimli öğrenme yöntemi önerilmektedir. Sadece $\% 20$ ile $\% 50$ arasında değişen etiketli örneklerden, önerilen yaklaşım ile denetimli eğitilen CNN' lerden potansiyel olarak daha iyi performans göstermektedir (Jing, Parag, Wu, Tian, \& Wang, 2021). UCF101 veri kümesi ile ön eğitimli modellerin kullanıldığı referans çalışmada, videoda yapılan spor aktivitesinin sınıflandırılması gerçekleştirilmiştir. $\mathrm{Bu}$ sayede, sporcuların kendilerine uygun antrenmanları bulmaları ve performanslarını artırmalarını hedefleyen bir çalışma yapılmıştır (Ramesh \& Mahesh, 2020). Bir diğer çalışmada ise videolar içerisinde yetişkin içeriği tespit edilmesine yönelik yapılan çalışmada video süresi boyunca belli periyotlarla insan cildi segmentasyonu yapılmıştır. İnsan cildi yoğunluğuna bakılarak içerik sınıflandırılması gerçekleştirilmiştir (Nugroho, Hardiyanto, \& Adji, 2015)

Kamu güvenlik kameralarından anomali (suç, kavga, trafik kazası vb.) tespit etmek için derin öğrenme kullanılabilir. Anomali tespitinde, bir anomaliyi tespit eden algoritma başka bir anomaliyi tespit etmekte başarısız olabilmektedir. Sistemin çevrimiçi çalışabilmesi için donanım ve yazılım (algoritma) birlikte uyumlu olarak tasarlanması gerekmektedir (Nayak, Pati, \& Das, 2021). Kapalı devre televizyon kamera sistemleri tarafından üretilen büyük verinin (big data) işlenmesiyle, ilgili sorunları çözmek için kamera konumu ve görüş alanı meta verilerini, mobil nesnelerin yörüngelerini ve video içerik analizi algoritmalarından (örneğin mobil nesnelerin tespiti ve hareketi) meta verileri birleştiren bir yaklaşım tanımlanmıştır. Bu metot ile araştırmacıların insan veya nesne içermeyen kameraların görüntüleri kaldırılarak, arama süresi kısaltılmaktadır (Panta, Qodseya, \& Péninou, 2018). Videolar üzerinde duman tespit edilmesi ile ilgili yapılan diğer bir çalışmada transfer öğrenmeden faydalanılmıştır. Videoda sabit kalan k1sımlar yerine hareket halinde olan yapıların görüntülerinden duman bilgisinin olup olmadığı derin öğrenme ile tespit edilmeye çalışılmıştır (Jia, ve diğerleri, 2021).

Videodan silah tespiti yapılan bir çalışmada ise başarısı kanıtlanmış modellerden VGG16 (Simonyan \& Zisserman, 2014), InceptionV3 (Szegedy, Vanhoucke, Ioffe, Shlens, \& Wojna, 2015), Inception-ResNetV2 (Szegedy, Ioffe, Vanhoucke, \& Alemi, 2016), SSDMobileNEtV1 (Howard, ve diğerleri, 2017), Faster RCNN (Ren, He, Girshick, \& Sun, 2016), YOLO V3 ve YOLO V4 (Bochkovskiy, Wang, \& Liao, 2020) algoritmaları kullanılarak gerçekleştirilen deneylerde, en performanslı modelin YOLO V4 olduğu görülmüştür. Transfer öğrenme için ImageNet (ImageNet, 2021) ve COCO (COCO, 2021) kullanılmışıtır. Bu çalışma ile video üzerinde gerçek zamanlı nesne tespiti yapılabileceği gösterilmiştir (Bhatti, Khan, Aslam, \& Fiaz, 2021).

\section{Derin Öğrenme ve Deneysel Çalışmalar}

\subsection{Veri Kümesi}

Tasarlanan sistemdeki her bir sınıfı belirlemek için fazla sayıda görüntüye ihtiyaç bulunmaktadır. Yine her bir sınıf için yaklaşık olarak aynı sayıda görüntü olması en ideal durumdur. $\mathrm{Bu}$ 
çalışmada, üç adet sınıf olacak şekilde tasarım yapılmıştır. $\mathrm{Bu}$ sınıflar tütün mamulleri, silah ve alkollü içeceklerdir. Yapılan araştırmalar sonucunda öncelikle Kaggle (Kaggle, 2021) internet sitesinde tütün mamulleri (Lee, 2019), silah (Sasank, 2019) ve alkollü içecekler (Nouisser, 2019) için veri kümeleri temin edilmiştir. Görsel olarak veri seti üzerinde yapılan incelemelerden sonra sınıflardaki resim sayıları şu şekilde olmuştur: tütün mamülleri sınıfı için 2147 resim, silah sınıfı için 728 resim ve alkollü içecekler sınıfı için 2928 resim. Özellikle, silah sınıfı için veri kümesinde görüntü sayısının yetersiz olmasından dolayı veri kümesini geliştirmeye çalışılmıştır. Bu amaçla öncelikle arama motorlarının görsel arama sonuçlarından faydalanılmıştır. Ayrıca Python programlama dilinde yazılmış "Bulk Bing Image Downloader" (Ostrolucky, 2020) uygulaması ile Bing arama motorunda verilen anahtar kelimelerimiz silah ve gun arama sonuçlarında gelen görüntülerin kaynak dosyaları elde edilmiştir.

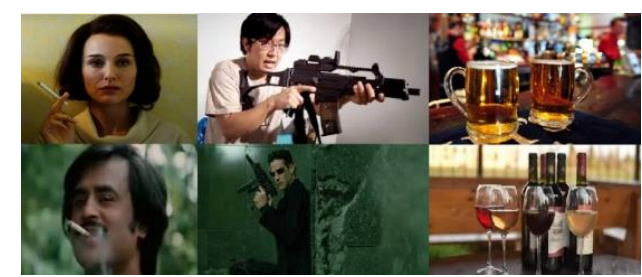

Şekil 1. Veri kümesinden örnek tütün mamulleri, silah ve alkollü içecek görüntüleri

\subsection{CNN Modeli ve Eğitimi}

Bu çalışmada, ilk olarak ağ yapısını bizim oluşturduğumuz model için Siralı (Sequential) metodu ile başlanmıştır. Modelimizin giriş katmanını oluşturacak ilk Conv2D (evrişim) katmanımızda 256 adet nöron ve $2 \times 2$ 'lik bir filtre tanımladıktan sonra $224 \times 224 \times 3$ olan giriş biçimimizi belirttik. Bu parametre ile sistemin eğitiminde ve eğitim sonrasında tahmin için modele verilecek resimlerin pikselleri bu tensöre dönüştürülmelidir. $\mathrm{Bu}$ katman için aktivasyon fonkiyonu "Relu" seçilmiştir. Sonrasında tanımlanan "Max Pooling2D" ile 2x2 ortaklama (pooling) işlemi yapılmakta ve girişe uygulanan resim verisi yarılanarak $111 \times 111 \times 256$ boyutuna indirilmektedir. Son olarak, batch normalizasyonu işlemi ile bu ara katman tamamlanmıştır.

Sonraki ara katman Conv2D' da 512 adet nöron ve $2 \times 2$ 'lik bir filtre tanımladıktan sonra aktivasyon fonksiyonu yine "relu" seçilmiştir. Sonrasında, tanımlanan "Max Pooling2D" ile 2x2 ortaklama işlemi yapılmakta ve girişe uygulanan resim verisi yarılanarak $55 \times 55 \times 512$ boyutuna indirilmektedir. Tekrar batch normalizasyonu işlemi ile bu ara katman tamamlanmış olur. Bundan sonraki 3 ara katmanda, sadece nöron sayısı değiştirilerek özellik çıkarma işlemine devam edilmiştir. Son aşamada ise 6x6×256' lük bir tensör kalmıştır. Flatten katmanı ile tensör bir vektöre dönüştürülürek özellik çıkarma işlemi tamamlanmış olmaktadır. Buradan sonraki ağ yapısı, sınıfları öğrenmek için geliştirilmektedir.

Dense katmanında (tam bağlı katman-fully connected layer) 256 nöron tanımlanmıştır. Aktivasyon fonksiyonu evrişim katmanlarında olduğu gibi "relu" seçilmiştir. Modelin, aşırı öğrenme (overfitting) sorununa takılmaması için 0,2 değerinde bir "Dropout" katmanı eklenmiştir. Son olarak, batch normalizasyonu işlemi ile bu katman da tamamlanmış olmaktadır.

Son olarak bir dense katmanı daha eklenmiştir ve bu katman 3 nörondan oluşur. $\mathrm{Bu}$ katmanın aktivasyon fonksiyonu "softmax" seçilerek modelin ağ yapısı tamamlanmış olur. Tasarlanan modelin eğitim işlemleri gerçekleştirilmiştir. Bu çalışma için Keras ve Tensorflow yapıları (frameworks) kullanılmıştır. Model olarak bir tane ağ yapısını oluşturduğumuz model ve transfer öğrenme metodu kullanılarak farklı modellerin başarımları ile çalışma gerçekleştirilmiştir.

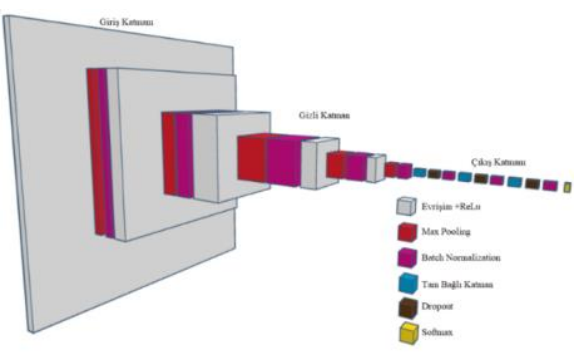

\section{Şekil 2. Oluşturulan CNN modelinin ăg katman yapısı}

A ̆g modelimiz üç ana kısımdan oluşmaktadır. İlk katman giriş katmanı modele eğitim ve tahmin için dışarıdan görüntü bilgisinin verildiği katmandır. İkinci katman ise gizli katman olarak isimlendirilmekte ve verilen görüntünün öznitelikleri bu katmanda çıkarılmaktadır. Son olarak, çıkış katmanında ise sınıfın öğrenildiği katman olarak görev yapmaktadır. Şekil 2' de gösterilen modelin dişında başarımı kanıtlanmış olarak ifade edilen modellerin giriş ve gizli katmanları dondurularak sadece çıkış katmanı ağının tekrar düzenlenmesi metodu ile transfer öğrenme gerçekleştirilmektedir. Transfer öğrenme bu başarısı kanıtlanmış modellerin özellik çıkartma katmanını kendi çalışmamızda kullanmamızı sağlamaktadır. Diğer bir fayda ise eğitilebilir parametre sayısının azalması ile eğitim süresi kısalmıştır.

\section{Deneysel Sonuçlar}

\subsection{CNN Model Eğitiminin Sonuçları}

Doğruluk model eğitimi sırasında modelin yaptığı doğru tahminlerin doğru ve yanlış tüm tahminlerin toplamına oranıdır. Kayıp ise modelin doğru çıkış değerinden ne kadar farklı tahmin değerinde bulunduğunu gösteren parametredir. Kayıp değeri ne kadar küçülürse model gerçek değere o kadar yakındır.

Ağını bizim oluşturduğumuz model \%86,75 doğruluk başarımı göstermiştir. Transfer öğrenme, veri kümesinin yetersiz olduğu durumlarda daha önceden çok büyük veri kümeleri ile eğitilmiş modellerin ağırlıklarını yani özellik çıkartma becerilerini kullanmamıza imkân vermektedir. Ön eğitimli modellerden MobileNetV2, DenseNet121, NasnetMobile, Resnet50V2 kullanılarak yazılımsal deneyler gerçekleştirilmiştir. Transfer öğrenmeden faydalanırken kullanılacak önceden eğitilmiş modelin çıkış katmanları model tasarımından çıkarılır ve katmanlar dondurularak üzerine bizim çıkış katmanlarımız eklenir. Eğitim az miktarda eğitilebilir parametre ile gerçekleştirilir. Bu durum, eğitimin daha kısa sürmesinin yanı sıra daha başarılı modeller elde edilmesini sağlamaktadır. İlk olarak, çıkıș katmanı için ağ yapısı ile ilgili denemeler yapılmıştır. Çıkış katmanında kullanılan katman sayısı ve nöron sayısı artırıldıkça başarım artmaktadır. Ancak eğitilmiş modelin boyutunun çok büyümesi tercih edilmemektedir. Ayrıca, bir noktadan sonra çıkış katmanlarının büyütülmesi iyi yönde değişime sebep olmamaktadır. Tablo 1'de bu durum kolayca görülmektedir. 
Tablo 1. Çıklş Katmanına Ă̆ Büyüklüğünün Etkisi

\begin{tabular}{|l|c|c|c|c|}
\hline \multirow{2}{*}{ Model Adı } & $\begin{array}{c}\text { Eğitilebilir Parametre } \\
\text { Sayısı }\end{array}$ & $\begin{array}{c}\text { Ĕ̆tim Başarım } \\
\mathbf{( \% )}\end{array}$ & $\begin{array}{c}\text { Doğrulama Başarım } \\
(\boldsymbol{\%})\end{array}$ & $\begin{array}{c}\text { Model Boyutu } \\
(\mathbf{M B})\end{array}$ \\
\hline \multirow{3}{*}{ MobileNetV2 } & 395.011 & 65,06 & 58,00 & 12 \\
\cline { 2 - 5 } & 593.155 & 65,34 & 50,35 & 14 \\
\cline { 2 - 5 } DenseNet121 & 2.762 .243 & 65,11 & 55,03 & 30 \\
\cline { 2 - 5 } & 329.475 & 73,62 & 74,74 & 32 \\
\cline { 2 - 5 } & 527.619 & 73,60 & 76,22 & 48 \\
\hline
\end{tabular}

Model eğitimi sırasında seçilen optimizasyon algoritmasının, kaç döngü (epoch) sayısında en iyi öğrenme düzeyine ulaşılacağını belirlemede önemli rol oynamaktadır. En iyi başarıma ulaşmak için optimize edici algoritmaların bazıları kayıp (loss) değerini az devir sayısında gürültü olușturarak düşürebilirken, bazıları da daha fazla devir sayısı ile daha az gürültü oluşturarak düşürebilirler. Ancak bilinmelidir ki devir sayısının fazla olması, başarımı sürekli yukarı doğru yükselten bir durum oluşturmamaktadır. Bir seviyeden sonra başarım düzeyi yatay eğri haline gelecektir.

Yapılan deneylerde farklı optimizasyon algoritmaları denenerek doğruluk ve kayı değerleri birlikte değerlendirildiğinde "Adam" ve "Rmsprop" optimizasyon algoritmalarının veri kümesi için uygun olduğuna karar verilmiştir.
Transfer öğrenme kullanılırken, modeller arasında ağ yapısının oluşturulması ve parametre sayılarında farklılıklar bulnmaktadır. Genellikle, daha fazla parametreye sahip modellerin daha başarılı olacağ düş̧ünülse de bu durum her zaman geçerli değildir. Tablo 1'den bu durum görülebilemektedir. Modeldeki hesaplanacak parametre sayısının artması, daha fazla işlem gücü gerektirecektir. Geliştirme yapılacak platformların işlem gücü göz önünde bulundurularak seçim yapılmalıdır. Yine, model dosyasının büyüklüğü önem arz etmektedir. Çünkü modelin çalışabilmesi için bulut ortamından veya yerel ortamdan hafızaya yüklenmesi gerekecektir. Büyük boyuttaki model dosyalarının yüklenmesi sistemin başlatılmasında gecikmelere sebep olabilmektedir. Bundan dolayı, küçük dosya boyutuna sahip modeller tercih edilmiştir. Bu çalışmada, veri kümemiz ile yapılan deneylerde ise Tablo 2'deki sonuçlar elde edilmiş̧ir.

Tablo 2: Ön eğitimli modellerin karşılaşttrılması

\begin{tabular}{|l|c|c|c|c|c|}
\hline \multirow{2}{*}{ Model } & \multicolumn{2}{|c|}{ Eğitim } & \multicolumn{2}{c|}{ Doğrulama } & \multirow{2}{*}{$\begin{array}{c}\text { Boyut } \\
\text { (MB) }\end{array}$} \\
\cline { 2 - 5 } & Doğruluk (\%) & Kayı & Doğruluk (\%) & Kayıp & 48 \\
\hline DenseNet121 & 0,74 & 0,62 & 0,64 & 0,73 & 39 \\
\hline NasnetMobile & 0,74 & 0,64 & 0,70 & 0,73 & 30 \\
\hline MobileNetV2 & 0,80 & 0,65 & 0,55 & 1,14 & 115 \\
\hline ResNet50V2 & 0,68 & 0,74 & 0,73 & 0,67 & 32 \\
\hline EfficientNetB0 & 0,90 & 0,26 & 0,13 & 0,95 & \\
\hline
\end{tabular}

Test edilen modellerden sadece "Efficient NetB0" farklı bir ağ yapısına sahiptir. Diğer modeller özellik çıkartma katmanları dondurularak aynı çıkış katmanı ve hiper parametreler kullanılarak eğitimler gerçekleştirilmiştir. Efficient NetB0 modeli en iyi başarımı göstermektedir. Aşıırı öğrenmeyi (overfitting) önlemek için ağda ki bazı nöronların unutmasını sağlamayan metot olan "drop out" yaklaşımının kullanılmasına rağmen sonuçlarda bir iyileşme olmamıştır. "ResNet50V2" modeli büyük

\section{Sonuç}

Bu çalışmada, derin öğrenme yaklaşımlarından CNN modeli geliştirilerek, iki farklı uygulamada çalıştırılmıştır. Modelin geliştirilmesi aşamasında iki metot benimsenmiştir. Birincisi, ağ yapısını oluşturduğumuz modelde farklı parametreler ile yapılan denemelerde ağ büyüklüğünün özellik çıkarma konusunda avantaj sağladığı görülmüştür. Yine çıkış katmanı için ağ e-ISSN: 2148-2683 dosya boyutuna sahip olmasından dolayı tercih edilmemiştir. Modellerin geliştirilen uygulamalarda çalıştırılabilmesi için dönüştürme işlemleri yapılması gerekmektedir. Bu dönüşümler sonucu hem tarayıcı hemde mobil uygulama için model dönüştürme işlemi sadece MobileNetV2 ile sorunsuz gerçekleștirildiği için uygulamalarda kullanılmak üzere seçilen model olmuştur.

büyüklüğünün artırılması olumlu sonuçlar vermiştir. Modelin geliştirilmesi için benimsenen diğer metot ise transfer öğrenmedir. Transfer öğrenme ile kullanılan başarısı kanıtlanmış modellerin özellik çıkarımı (feature extraction) konusunda başarılı olduğu gözlenmiştir. Hazırlanan farklı çıkış katmanları ile yapılan deneylerde, ağ yapısını olușturduğumuz modelden daha başarılı olunduğu görülmüşşür. Geliştirilen tarayıcı eklentisinde modelin depolanmasının, Google Firebase "Storage" bulut 
hizmetinde yapılması kullanım ve güncelleme kolaylığı sağlamaktadır. $\mathrm{Bu}$ çalışmanın devamı olarak, videoların görüntüden sınıflandırması yapılmadan ilk olarak videonun meta verilerine bakarak bir sınıflandırma işlemi uygulanması ek bir önlem olarak sistemin performansını artıracağı düşünülmektedir.

\section{Kaynakça}

(2021). Kaggle: https://www.kaggle.com/ adresinden alınd1 (2021). ImageNet: https://image-net.org/ adresinden alınd1 (2021). COCO: https://cocodataset.org/ adresinden alınd1

Abu-El-Haija, S., Kothari, N., Lee, J., Natsev, P., Toderici, G., Varadarajan, B., \& Vijayanarasimhan, S. (2016, 9 27). YouTube-8M: A Large-Scale Video Classification Benchmark. arxiv.org: https://arxiv.org/abs/1609.08675 adresinden alınd 1

Bhatti, M. T., Khan, M. G., Aslam, M., \& Fiaz, M. J. (2021, 2 12). Weapon Detection in Real-Time CCTV Videos Using Deep Learning. IEEE Access, s. 34366 - 34382. doi:10.1109/ACCESS.2021.3059170

Bochkovskiy, A., Wang, C.-Y., \& Liao, H.-Y. M. (2020, 4 23). YOLOv4: Optimal Speed and Accuracy of Object Detection. arxiv.org: https://arxiv.org/abs/2004.10934 adresinden alınd1

Chen, Y.-L., Chang, C.-L., \& Yeh, C.-S. (2017, Eylül). Emotion classification of YouTube videos. Decision Support Systems, s. 40-50. doi:10.1016/j.dss.2017.05.014

Hammam, A. M. (2019). An Extensible, Modular Framework for Classifying YouTube Videos Using Web and Social Media. 2019 IEEE Conference on Multimedia Information Processing and Retrieval (MIPR). IEEE. doi:10.1109/MIPR.2019.00092

Howard, A. G., Zhu, M., Chen, B., Kalenichenko, D., Wang, W., Weyand, T., ... Adam, H. (2017, 4 17). MobileNets: Efficient Convolutional Neural Networks for Mobile Vision Applications. arxiv.org: https://arxiv.org/pdf/1704.04861.pdf adresinden alınd 1

Jia, C., Wang, S., Zhang, X., Wang, S., Liu, J., Pu, S., \& Ma, S. (2019). Content-Aware Convolutional Neural Network for In-Loop Filtering in High Efficiency Video Coding. IEEE Transactions on Image Processing, 3343-3356. doi:10.1109/TIP.2019.2896489

Jia, Y., Chen, W., Yang, M., Wang, L., Liu, D., \& Zhang, Q. (2021). Video smoke detection with domain knowledge and transfer learning from deep convolutional neural networks. Optik(240). doi:10.1016/j.ijleo.2021.166947

Jing, L., Parag, T., Wu, Z., Tian, Y., \& Wang, H. (2021). VideoSSL: Semi-Supervised Learning for Video Classification. Winter Conference on Applications of Computer Vision (WACV) (s. 1110-1119). IEEE/CVF.

Karpathy, A., Toderici, G., Shetty, S., Leung, T., Sukthankar, R., \& Fei-Fei, L. (2014). Large-Scale Video Classification with Convolutional Neural Networks. 2014 IEEE Conference on Computer Vision and Pattern Recognition. IEEE. doi:10.1109/CVPR.2014.223

LeCun, Y., Bengio, Y., \& Hinton, G. (2015). Deep learning. Nature, 436-444. doi:10.1038/nature14539

Lee, H. (2019). Cigarette Smoker Detection. 04 24, 2021 tarihinde https://www.kaggle.com/vitaminc/cigarette-smokerdetection adresinden alındı

Nayak, R., Pati, U. C., \& Das, S. K. (2021). A comprehensive review on deep learning-based methods for video anomaly detection. Image and Vision Computing. doi:10.1016/j.imavis.2020.104078

Nouisser, K. (2019). Alcoholic Drinks. 04 24, 2021 tarihinde https://www.kaggle.com/khalilnouisser/alcoolicdrinks5 adresinden alındı

Nugroho, H. A., Hardiyanto, D., \& Adji, T. B. (2015). Negative content filtering for video application. 7th International Conference on Information Technology and Electrical Engineering (ICITEE). IEEE. doi:10.1109/ICITEED.2015.7408912

Ostrolucky, G. (2020). Bulk Bing Image Downloader. 04 24, 2021 tarihinde https://github.com/ostrolucky/Bulk-Bing-Imagedownloader adresinden alınd 1

Panta, F. J., Qodseya, M., \& Péninou, A. (2018). Management of Mobile Objects Location for Video Content Filtering. MoMM2018: Proceedings of the 16th International Conference on Advances in Mobile Computing and Multimedia (s. 44-52). New York: Association for Computing Machinery. doi:10.1145/3282353.3282368

Ramesh, M., \& Mahesh, K. (2020). A Performance Analysis of Pre-trained Neural Network and Design of CNN for Sports Video Classification. International Conference on Communication and Signal Processing. Chennai: IEEE. doi:10.1109/ICCSP48568.2020.9182113

Ren, S., He, K., Girshick, R., \& Sun, J. (2016, 1 6). Faster RCNN: Towards Real-Time Object Detection with Region Proposal Networks . arxiv.org: https://arxiv.org/pdf/1506.01497v3.pdf adresinden alındı

Sasank, S. (2019). Guns Object Detection. 04 24, 2021 tarihinde https:/www.kaggle.com/issaisasank/guns-object-detection adresinden alınd 1

Shaout, A., \& Crispi, B. (2020). Streaming Video ClassificationUsing Machine Learning. The International Arab Journal of Information Technology, 667-682. doi:10.34028/iajit/17/4A/13

Simonyan, K., \& Zisserman, A. (2014, 9 4). Very Deep Convolutional Networksfor Large-Scale Image Recognition. arXiv.org: https://arxiv.org/abs/1409.1556 adresinden alındı

Szegedy, C., Ioffe, S., Vanhoucke, V., \& Alemi, A. (2016, 2 23). Inception-v4, Inception-ResNet and the Impact of Residual Connections on Learning. arXiv.org: https://arxiv.org/abs/1602.07261v2 adresinden alınd1

Szegedy, C., Vanhoucke, V., Ioffe, S., Shlens, J., \& Wojna, Z. (2015, 12 5). Rethinking the Inception Architecture for Computer Vision. arXiv.org: https://arxiv.org/abs/1512.00567v3 adresinden alınd1

Wu, Z., Yao, T., Fu, Y., \& Jiang, Y.-G. (2017). Deep learning for video classification and captioning. Frontiers of Multimedia Research, 3-29. doi:10.1145/3122865.3122867 